\title{
LINK AND PATH ASYMMETRY ISSUES IN IP OVER WDM TRANSPORT NETWORKS
}

\author{
D.A. Schupke \\ Munich University of Technology \\ Institute of Communication Networks \\ 80290 Munich, Germany \\ Tel.: +4989 289-23511, Fax: +4989 289-63511 \\ Dominic.Schupke@lkn.ei.tum.de
}

\begin{abstract}
In this paper we consider link and path asymmetry in IP over WDM transport networks. While transport networks are traditionally symmetric the carried Internet traffic is very asymmetric involving a potential for major network cost reductions. We determine where asymmetry can be introduced and indicate necessary protocol adaptations. We raise investigation issues in asymmetry which need to be pursued in future.
\end{abstract}

\section{INTRODUCTION}

Measurements in the Internet show a highly asymmetric characteristic of the traffic flow [1, 2]. One of the reasons for this is that a large portion originates from servers connected to the Internet, which tend to involve much more generated traffic (information supply) than received traffic (requests, etc.) With the currently emerging Internet applications, it is expected that this characteristic will remain for the future.

Today in transport networks traffic is carried over symmetric links with protocols like SDH/SONET or point-to-point Gigabit Ethernet which in turn are operated over WDM networks. Therefore one transmission direction is underutilized by the asymmetric Internet traffic. Since optical line port equipment (in particular the lasers) is still a big cost factor, cost reductions are expected when introducing asymmetric links in the network. 
Furthermore in transport networks routing is done symmetrically, e.g. a bidirectional path traverses the same nodes for both directions. This can result in inefficient usage of the network resources.

In the following we classify the different possibilities for link and path asymmetry. We discuss realizations of asymmetry in IP over WDM networks and indicate necessary protocol adaptations. In conclusion we raise the investigation issues in asymmetry which need to be pursued in future.

\section{ASYMMETRY IN IP OVER WDM NETWORKS}

We consider links and paths on both the IP layer and the WDM layer. Paths on the WDM layer represent (virtual) links seen by the IP layer. Dealing with future high traffic volumes in transport networks we do not consider multiplexing of layers between IP and WDM (which can provide links and paths by themselves).

There are both unidirectional (simplex) or bidirectional (in this context full-duplex) links and paths possible. On the WDM layer paths are also called lightpaths [3]. Link (path) symmetry means that a bidirectional link (path) has the same characteristic in each direction. Then asymmetry can occur in different fashions:

- IP and WDM layer: Two nodes communicate in one direction only (unidirectionality).

- IP and WDM layer: Different nodes are traversed by the two unidirectional paths for each direction which together provide a bidirectional connectivity between two nodes (path asymmetry).

- WDM layer: On a segment the wavelengths of a bidirectional lightpath are different for each direction (wavelength asymmetry).

- IP layer: The bandwidth capacity is different between each direction of a bidirectional link (bandwidth asymmetry). Thus unidirectionality is a special case of bandwidth asymmetry.

- WDM layer: The number of lightpaths provided to an endpoint pair on the IP layer differs between both directions. This translates to bandwidth asymmetry seen by the IP layer.

Asymmetry also occurs with multicasting and protection schemes (e.g. one sided protection switching), which is not covered here.

From the network management point of view asymmetry introduces more effort in network configuration and in the administration of network data. Therefore for each of these different possibilities the efficiency gain 
has to be weighted against the increase of network management complexity.

\section{UNIDIRECTIONALITY}

In IP networks to make two routers adjacent a bidirectional link connecting them is required. The support for unidirectional links has been motivated by IP over broadcast satellite networks and is equally applicable in high bandwidth IP over WDM networks. A mechanism to emulate bidirectional connectivity over a unidirectional link at the link layer is proposed in [4], and can be used as illustrated in figure 1.

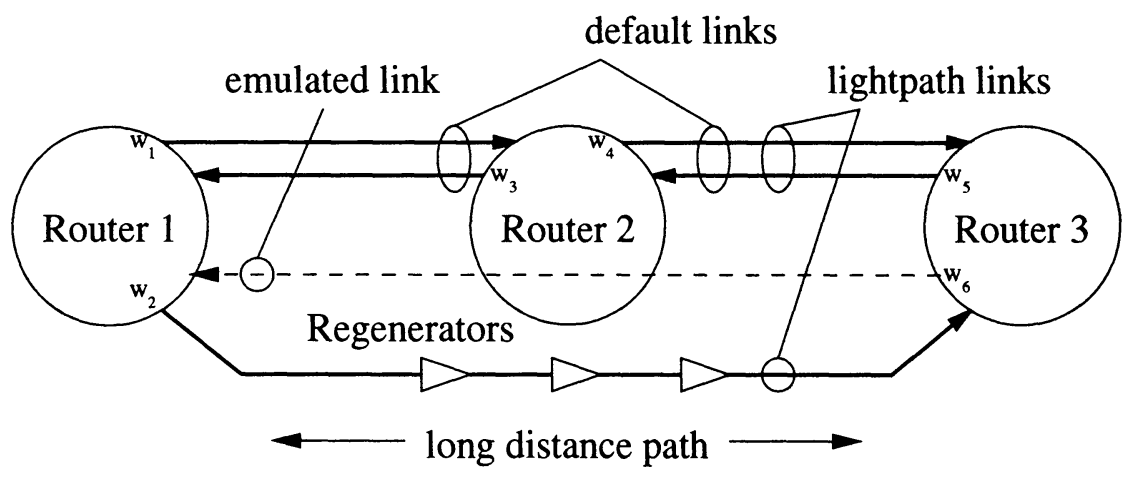

Figure 1: A unidirectional link with a tunnel as proposed in [4].

The bidirectional default connections are provided from router 2 to routers 1 and 3 . If there is a high traffic demand from router 1 to 3 but not from router 3 to 1 , it becomes desirable that a unidirectional (virtual) link is established between them (short-cut). As the IP layer needs to see a bidirectional link, by the extension in [4] a unidirectional tunnel is established from router 3 to 1 operated over the default links and router 2 .

In comparison to a bidirectional link, a sending unit at router 3 was saved, e.g. a Packet over SDH/SONET (POS) interface not equipped with a laser.

The physical distance between routers 1 and 3 can become very long requiring expensive regenerators to refresh the signal. A bidirectional link needs regenerators for each direction. In comparison, the unidirectional constellation needs only half of the regenerators of a bidirectional link, since the return link is provided over an existing path.

In this sense unidirectionality generally requires less resources in the WDM network than bidirectionality. This also holds for networks with 
nodes having partial wavelength conversion. Reducing the number of lightpaths in effect reduces the number of potentially needed converters.

Therefore with unidirectional links the costs introduced by regenerators and wavelength converters can be kept at minimum.

One important question is the allocation of the IP routing weights. Referring again to figure 1 , we can provide an asymmetric setting of $w_{2} \leq w_{1}+w_{4}$ to let the traffic flow over the short-cut and $w_{6}>w_{3}+w_{5}$ to reduce the tunneling effort. As these weights can have an impact on network-wide routing, this technique has to be considered when allocating the weights in the whole network (see also section 5).

We note here that establishing undirectional lightpaths should be possible even in WDM networks operating with automated lightpath set-up, e.g. provided by a MPLS control plane (so-called MP $\lambda S$ [5]). Paths in MPLS are by definition unidirectional. There are proposals for the extension of MPLS to directly set up bidirectional paths in optical networks [6], mainly to avoid contention problems. These extensions can coexist with unidirectional path set-up.

\section{PATH AND WAVELENGTH ASYMMETRY}

Firstly we consider wavelength asymmetry in bidirectional paths. Figure 2 depicts the situation of a four node star network [3]. Assume that the nodes are optical cross-connects without wavelength converters interconnected by a bidirectional fiber pair and sources can choose from two wavelengths in the system.

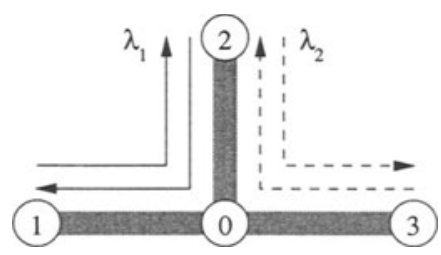

(a)

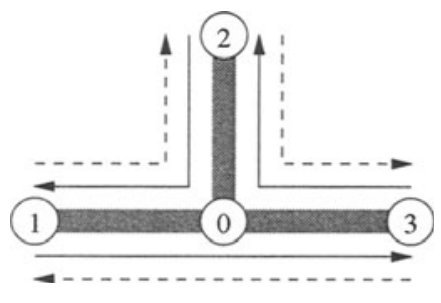

(b)

Figure 2: A star network with (a) wavelength symmetry and (b) with wavelength asymmetry of paths.

A full mesh demand of one bidirectional path between the nodes 1,2 and 3 should be provided. With wavelength symmetry in figure 2 (a) only 2 out of 3 bidirectional paths can be provided, whereas the asymmetric assignment in figure 2 (b) can provide for the full demand. 
Since wavelength selection seems not to be a crucial point for the routing process [7], wavelength asymmetry can become feasible for WDM networks. For example in MP $\lambda \mathrm{S}$ the wavelength assignment on a link is likely to be done by the signaling protocol for each direction independently.

Another example is the converterless WDM bidirectional line-switched ring using two fibers (BLSR/2) [3] which also requires wavelength asymmetry. Such a BLSR/2 consists of two counterrotating (and unidirectional) rings. One half of the wavelengths is used for working traffic, and the other half for protection. To avoid wavelength converters, two complementary sets of wavelengths are assigned to both rings. The working wavelengths of one ring are the protection wavelengths of the other. As bidirectional paths are routed symmetrically on the ring, different wavelengths are needed for each direction.

Path asymmetry, however, is highly controversial, because it makes routing information more unmanageable. Path asymmetry in WDM networks comes to front when introducing partial wavelength conversion in the network. Figure 3 (a) depicts the situation of a grid-like network with four nodes and existing lightpaths. Assume that the nodes are optical cross-connects each having a single wavelength converter available. The cross-connects are interconnected again by bidirectional fiber pairs and sources can select one of two wavelengths in the system.

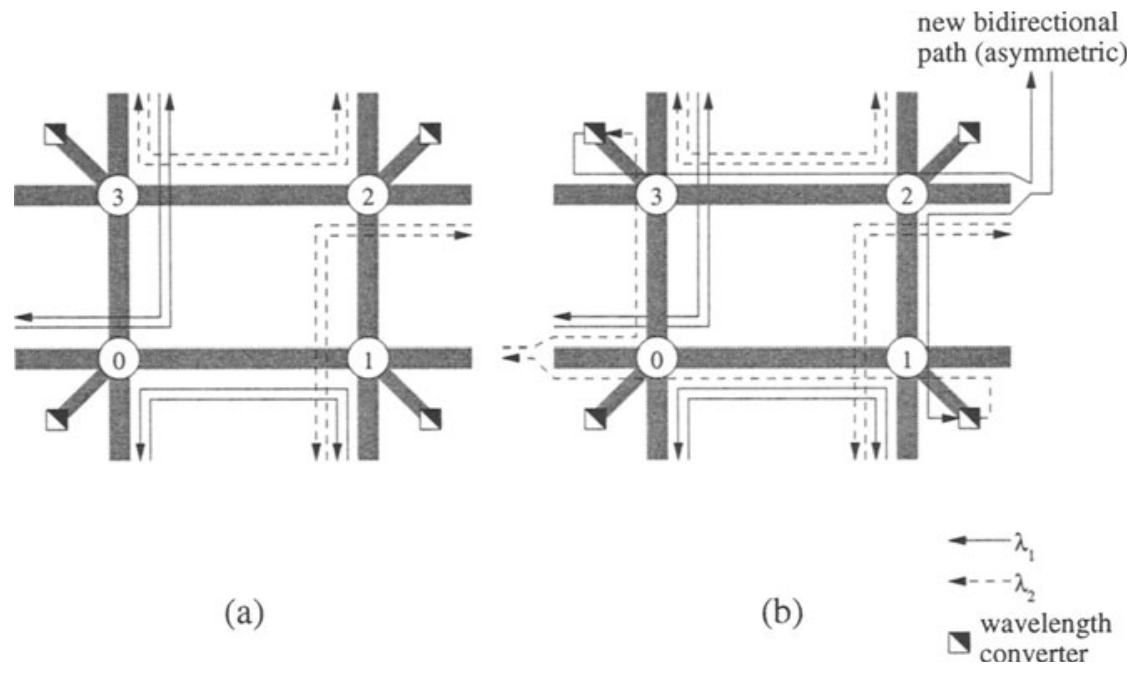

Figure 3: (a) A network with existing lightpaths. (b) A new bidirectional lightpath demand routed asymmetrically. 
Consider the following scenario. A new bidirectional lightpath between nodes 0 and 2 has to be provided without changing the existing lightpaths. This can only be done by an asymmetric path as depicted in figure $3(b)$.

For WDM networks with partial wavelength conversion asymmetric paths introduce a further degree of freedom which results in efficient network utilization. Thus it becomes an investigation issue what can be gained from path asymmetry in WDM networks.

On the IP layer nodes are operated with distributed routing protocols like OSPF, which relies on routing weights assigned to links. On one hand different weight assignments for both directions of each link in a network do not necessarily result in path asymmetry.

On the other hand even when assigning equal weights for both directions of every link in the network we can obtain path asymmetry when there is more than one shortest path between two nodes. Depending on the implementation of the routing protocol, the nodes can choose different paths for the two directions of a connectivity, since the paths are evaluated as equally "shortest." This last issue has been avoided so far by dimensioning the routing weights such that unique shortest paths exist between the node pairs $[8,9]$.

In networks with IP as a connectionless protocol packets can take an arbitrary route, but in IP backbones the network service operators have dimensioned the routing weights in such a way to obtain predictability of the traffic flow. Thus it becomes an issue how to assign the weights such that IP path symmetry including unidirectional and parallel links (see below) is achieved and traffic flow predictability is still assured.

\section{BANDWIDTH ASYMMETRY}

Firstly we consider the case where the bandwidths are different in the two directions of a bidirectional lightpath. We assume that line ports become more expensive with growing bitrate. For POS point-to-point systems there is no reason against having an asymmetric bandwidth allocation as long as bidirectional communication for IP is possible. The POS protocol specification, however, has to be modified slightly for this.

Consider a point-to-point transmission with traffic of $10 \mathrm{~Gb} / \mathrm{s}$ in one and $2.5 \mathrm{~Gb} / \mathrm{s}$ in the other direction which should be operated with POS as link layer (asymmetry ratio of 4:1). With today's equipment two STM64 ports are needed where one direction is utilized by $25 \%$ only. Assume half of the cost of the two ports to provide for each direction of the link. Assume furthermore that the cost of a STM-N system doubles as N quadruplicates. Then an asymmetric $10 \mathrm{~Gb} / \mathrm{s} \uparrow-2.5 \mathrm{~Gb} / \mathrm{s} \downarrow$ system would 
be $75 \%$ of the costs of the symmetric STM- 64 system. Thus again we obtain cost reductions for these links.

An important bandwidth asymmetry also occurs when two routers are connected bidirectionally by lightpaths, but with a different number of lightpaths for each direction [2], see figure 4. The advantage of this configuration is again that we can economize on sending and regenerating equipment.

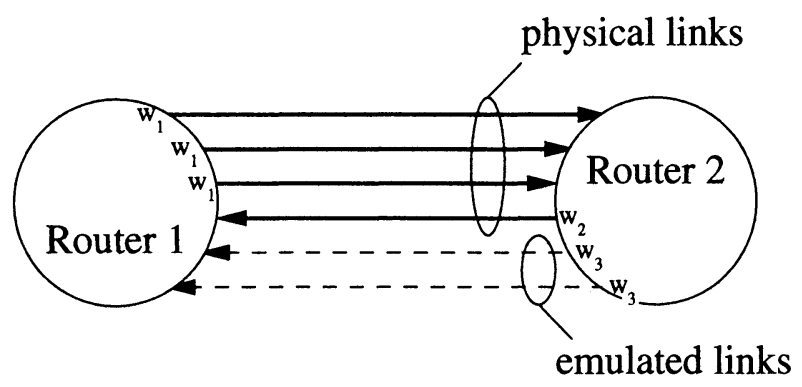

Figure 4: Bandwidth asymmetry by different lightpath allocations for each direction and a tunnel as in [4].

There are three possibilities to handle this case at the interface WDM/IP:

1 The link layer provides a virtual interface (with asymmetric bandwidth) and performs inverse multiplexing to split the data on the lightpaths. A major problem becomes chromatic dispersion causing that the signals on the lightpaths experience different delays. Thus synchronization on the receiver side becomes too difficult.

2 The mechanism in [4] again provides a solution. Referring to figure 4 there is one "physical" and two emulated bidirectional links between router 1 and 2 . Thus the IP layer sees the parallel links and uses them simultaneously if at router 1 the weights are equal (at router 2: $w_{2}<w_{3}$.)

3 Another possibility is that the IP layer sees the parallel links provided by the mechanism in [4] and distributes the traffic depending on some classification, e.g. flow classification or QoS class. Thus a router with traffic engineering capabilities is required.

For the possibility 2 again the setting of the weights in the whole network becomes an issue. Moreover as chromatic dispersion is present, packets on one lightpath can overtake packets on other lightpaths. If these packets belong to the same TCP flow, the performance is reduced, since 
the packets are not in order [10]. This issue has been addressed in [11], where solutions are presented to evenly distribute flows on parallel links (which occur to be equal-weight paths).

\section{CONCLUSIONS}

For IP over WDM networks we made the classification of asymmetry in unidirectionality, path asymmetry, wavelength asymmetry and bandwidth asymmetry. We presented different realizations to provide asymmetry in these networks on a link and path basis.

We have shown where investment cost reductions are obtained when asymmetric links and paths are employed in the network. However, higher network management effort and partially also protocol changes are introduced. Therefore we propose further considerations to determine what kind of asymmetry can help to reduce the overall costs of a network. A quantitative analysis of the advantages of asymmetry is performed in future investigations.

Unidirectional and parallel links are likely to be gradually employed in IP networks. The routing weight assignment problem has to be reconsidered to allow for unidirectional links and multiple paths between router-pairs.

\section{ACKNOWLEDGMENTS}

This work was supported by the German Federal Ministry of Education and Research (BMBF) in the project TransiNet (http://www. transinet.de/).

The author would like to thank A. Autenrieth for his helpful discussions. 


\section{References}

[1] K. Thompson, G.J. Miller, and R. Wilder. Wide-area Internet traffic patterns and characteristics. IEEE Network, 11(6), 1997.

[2] B.S. Arnaud. Architectural and Engineering Issues for Building an Optical Internet. In All-Optical Networking: Architecture, Control, and Management Issues. SPIE, 1998.

[3] R. Ramaswami and K.N. Sivarajan. Optical Networks: A Practical Perspective. Morgan Kaufmann Publishers, 1998.

[4] E. Duros, W. Dabbous, Izumiyama H., N. Fujii, and Y. Zhang. A Link Layer Tunneling Mechanism for Unidirectional Links. InternetDraft draft-ietf-udlr-1ltunnel-04, 2000.

[5] D.O. Awduche, Y. Rekhter, J. Drake, and R. Coltun. MultiProtocol Lambda Switching: Combining MPLS Traffic Engineering Control With Optical Crossconnects. Internet-Draft draft-awduche-mpls-te-optical-02, 2000.

[6] D. Guo, L. Zhang, J. Fu, and R. Cheung. Extensions to RSVP-TE for Bi-directional Optical Path Setup. Internet-Draft draft-sorrento-rsvp-bi-osp-00, 2000.

[7] H. Zang, J.P. Jue, and B. Mukherjee. A review of routing and wavelength assignment approaches for wavelength-routed optical WDM networks. Optical Networks Magazine, Jan 2000.

[8] A. Bley, M. Grötschel, and R. Wessäly. Design of Broadband Virtual Private Networks: Model and Heuristics for the B-WiN. Technical report, Konrad-Zuse-Zentrum für Informationstechnik Berlin, 1998.

[9] W.B. Ameur, E. Gourdin, and B. Liau. Internet Routing an Topology Problems. In Second International Workshop on the Design of Reliable Communication Networks (DRCN), 2000.

[10] C. Huitema. Routing in the Internet. Prentice-Hall, 2000. 
[11] D. Thaler and C. Hopps. Multipath Issues in Unicast and Multicast Next-Hop Selection. Internet-Draft draft-thaler-multipath-05, 2000 . 